\section{Aerobic Oxidation of Benzyl- and Allylic Alcohols under Visible Light Irradiation of a Fluorescent Lamp in the Presence of Catalytic Iodine}

\author{
Hiroki NAKAYAMA and Akichika ITOH* \\ Gifu Pharmaceutical University; Mitahora-higashi, Gifu 502-8585, \\ Japan. Received August 11, 2006; accepted September 16, 2006
}

\begin{abstract}
Benzyl alcohols and allylic alcohols were found to be oxidized to the corresponding aldehydes in the presence of a catalytic amount of iodine under irradiation of a fluorescent lamp.
\end{abstract}

Key words aerobic oxidation; aldehyde; allyl alcohol; benzyl alcohol; catalytic iodine

Aldehydes are important intermediates for many chemical products, and synthetic methods of these compounds have been developed from the viewpoint of both oxidation and reduction for a long time. Although a variety of methods for oxidation of alcohols to aldehydes, especially, have been extensively developed, these methods are sometimes problematic because many reagents or heavy metals of environmentally high impact are required. ${ }^{1)}$ Regarding benzylic alcohols and allylic alcohols, in contrast to the simple alkanol, they have proved to be effectively oxidized to the corresponding aldehydes or ketones with active $\mathrm{MnO}_{2}$; however, there are some problems due to variation of the activity of $\mathrm{MnO}_{2}$ according to the way of pretreatment and the requirement of a large amount. Swern oxidation, which is a typical oxidation using no heavy metals, has been used as a mild synthetic method of aldehydes from alcohols; however, this procedure is tedious, and halogenated solvents of environmentally high impact and a large amount of several reagents such as DMSO and oxalyl chloride, which are not suitable for a large-scale reaction, are required. On the other hand, recently, catalytic oxidation processes with molecular oxygen, which has received much attention as an ultimate oxidant and generate little waste, have been reported by many researchers. ${ }^{2-11)}$ With this background in mind, we have previously reported oxidation of benzylic alcohols and allylic alcohols to provide the corresponding benzaldehydes and $\alpha, \beta$-unsaturated aldehydes with a stoichiometric amount of iodine under UV-irradiation conditions. ${ }^{12)}$ In the course of our further study of this reaction condition, we have found that this oxidation proceeds even under irradiation of visible light irradiated from a general-purpose fluorescent lamp in the presence of catalytic iodine. ${ }^{13-16)}$ In this letter, we report our study of the scope and limitations of this new non-metal oxidation method (Chart 1).

Table 1 shows the results of a study of reaction conditions conducted with 4-tert-butylbenzylalcohol (1) as a test substrate under the conditions of 10- or 24-h external irradiation by a general-purpose fluorescent lamp in the presence of a catalytic amount of iodine in an oxygen atmosphere. ${ }^{17,18)}$ Among the solvents examined, ethyl acetate was found to most efficiently afford 4-tert-butylbenzaldehyde (2) (entries $1-5)$. We were surprised to find that the yield of $\mathbf{2}$ was gradually reduced when the amount of iodine was increased (en-

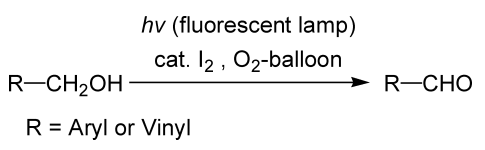

Chart 1

Table 1. Study of Reaction Conditions of Aerobic Photooxidation

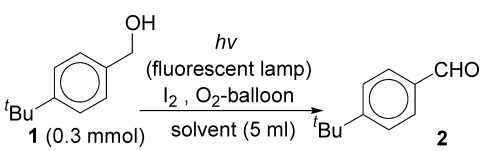

\begin{tabular}{|c|c|c|c|c|}
\hline Entry & $\mathrm{I}_{2}(\mathrm{eq})$ & Solvent & $t(\mathrm{~h})$ & Yield of $2(\%)^{a}$ \\
\hline 1 & 0.1 & Acetone & 10 & 36 \\
\hline 2 & 0.1 & $\mathrm{CH}_{3} \mathrm{CN}$ & 10 & Trace \\
\hline 3 & 0.1 & Hexane & 10 & Trace \\
\hline 4 & 0.1 & $i \mathrm{Pr}_{2} \mathrm{O}$ & 10 & 18 \\
\hline 5 & 0.1 & EtOAc & 10 & 61 \\
\hline 6 & 0.1 & EtOAc & 24 & 87 \\
\hline 7 & 0.1 & EtOAc & 24 & $0^{b)}$ \\
\hline 8 & 0.1 & EtOAc & 24 & Trace $^{c)}$ \\
\hline 9 & 0.2 & EtOAc & 10 & 58 \\
\hline 10 & 0.3 & EtOAc & 10 & 37 \\
\hline 11 & 0.4 & EtOAc & 10 & Trace \\
\hline 12 & - & EtOAc & 24 & 0 \\
\hline
\end{tabular}

a) The yields are for pure, isolated products. b) The reaction was carried out in the dark. c) The reaction was carried out in Ar atmosphere.

tries $5,9-11)$. The reason is not clear yet; however, we believe that decrease of the transmission of light due to an increase of the dissolving iodine is cause. That no oxidation proceeded without either irradiation by a fluorescent lamp or the addition of iodine shows the necessity of both for this reaction (entries 7, 12). Furthermore, from the fact that yields of the target substance were reduced substantially when conducting the reaction under the flow of argon, we can assume that the actual oxidant in this reaction is molecular oxygen (entry 8).

Table 2 shows the scope and limitations of this oxidation reaction using a variety of alcohols. Benzyl alcohols, in general, afford the corresponding benzaldehydes in high yields regardless of an electron-donating group or electron-withdrawing group at the aromatic nucleus, and primary or secondary alcohols; however, the reactivity of $\mathbf{5}$ was found to be different from others ${ }^{19)}$ (entries 1-7). Cinnamyl alcohol (15), which is an allylic alcohol, afforded cinnamaldehyde (16) in $56 \%$ yield due to generation of several unidentified by-products. On the other hand, 3-pyridinemethanol (17), which is a heterocyclic alcohol, afforded the corresponding aldehyde 18 in a trace amount and most of 17 was recovered. Furthermore, dodecanol (19), an aliphatic alcohol, was intact, and the starting material was recovered quantitatively (entry 10).

Chart 2 shows a possible path of this oxidation, which is postulated by considering the necessity of continuous irradiation, ${ }^{20)}$ a catalytic amount of iodine and molecular oxygen in this reaction. The radical species $\mathbf{2 0}$ is thought to be generated by abstraction of a hydrogen radical with an iodo radical, formed by irradiation of iodine (Eqs. 1, 2). Iodine, then, was formed by aerobic photo-oxidation of hydrogen iodide, which is generated in Eq. 2 (Eq. 3). Aldehyde 21 was af- 
Table 2. Aerobic Photooxidation for Various Alcohol Substrates substrate
$(0.3 \mathrm{mmol})$$\stackrel{\begin{array}{c}h v \text { (fluorescent lamp) } \\ \mathrm{I}_{2}(0.1 \text { equiv. }), \mathrm{O}_{2} \text {-balloon }\end{array}}{\text { EtOAc }(5 \mathrm{ml}), 24 \mathrm{~h}}$ product

Entry

a) The yields are for pure, isolated products. b) Most of $\mathbf{1 7}$ was recovered. c) The starting material 19 was recovered quantitatively.

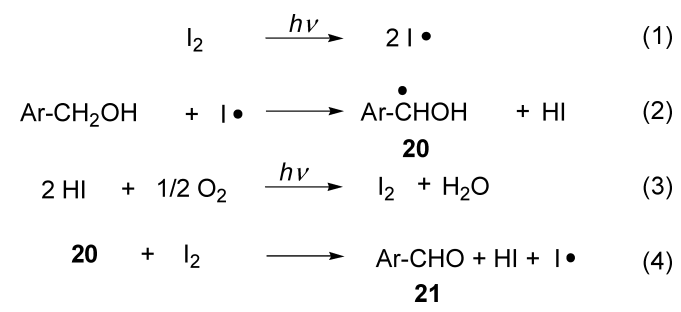

Chart 2. Plausible Path of the Aerobic Photo-oxidation of Alcohols

forded by abstraction of a hydrogen radical with iodine (Eq. $4)^{21)}$ In the case of $\mathbf{1 7}$, pyridine ring is assumed to trap a hydrogen iodide, and the subsequent reaction, therefore, did not proceed.
In conclusion, this new method for the preparation of benzaldehydes and $\alpha, \beta$-unsaturated aldehydes is convenient in the viewpoint of using visible light irradiated from a generalpurpose fluorescent lamp instead of UV light from a highpressure mercury lamp, and is thought to be environmentally benign, due to all the factors of the use of molecular oxygen as terminal oxidant, EtOAc of environmentally low impact as solvent, catalytic amount of iodine and no metals. The study of scale-up of this reaction is now in progress in our laboratory.

\section{References and Notes}

1) "Comprehensive Organic Transformations: A Guide to Functional Group Preparations," ed. by Larock R. C., Wiley-VCH, New York, 1999.

2) Ohkubo K., Suga K., Fukuzumi S., Chem. Commun., 2006, 20182020 (2006).

3) Guan B., Xing D., Cai G., Wan X., Yu N., Fang Z., Yang L., Shi Z., J. Am. Chem. Soc., 127, 18004-18005 (2005).

4) Mu R., Liu Z., Yang Z., Liu Z., Wu L., Liu Z.-L., Adv. Synth. Catal., 347, 1333-1336 (2005).

5) Schultz M. J., Hamilton S. S., Jensen D. R., Sigman M. S., J. Org. Chem., 70, 3343-3352 (2005).

6) Liu R., Liang X., Dong C., Hu X., J. Am. Chem. Soc., 126, $4112-$ 4113 (2004).

7) Mori K., Hara T., Mizugaki T., Ebitani K., Kaneda K., J. Am. Chem. Soc., 126, 10657-10666 (2004).

8) Marko I. E., Gautier A., Dumeunier R., Doda K., Philippart F., Brown S. M., Urch C. J., Angew. Chem. Int. Ed., 43, 1588-1591 (2004).

9) Stahl S. S., Angew. Chem. Int. Ed., 43, 3400-3420 (2004), and references cited therein

10) Iwasawa T., Tokunaga M., Obora Y., Tsuji Y., J. Am. Chem. Soc., 126, $6554-6555$ (2004).

11) Jensen D. R., Schultz M. J., Mueller J. A., Sigman M. S., Angew. Chem. Int. Ed., 42, 3810-3813 (2003).

12) Itoh A., Kodama T., Masaki Y., Chem. Lett., 2001, 686-687 (2001).

13) Pavlinac J., Zupan M., Stavber S., J. Org. Chem., 71, 1027-1032 (2006).

14) Mori N., Togo H., Tetrahedron, 61, 5915-5925 (2005).

15) Mori N., Togo H., Synlett, 2004, 880-882 (2004).

16) Miller R. A., Hoerrner R. S., Org. Lett., 5, 285-287 (2003).

17) A typical procedure follows: A dry ethyl acetate solution $(5 \mathrm{ml})$ of the 4-tert-butylbenzylalcohol $(0.3 \mathrm{mmol})$ and $\mathrm{I}_{2}(0.03 \mathrm{mmol})$ in a Pyrex test tube equipped with an $\mathrm{O}_{2}$-balloon, was stirred and irradiated at room temperature for $24 \mathrm{~h}$ with four of $22-\mathrm{W}$ fluorescent lamps, which was set from the test tube in the distance of $80 \mathrm{~mm}$. The reaction mixture was concentrated under reduced pressure, and dissolved in diethyl ether, and washed with sodium thiosulfate and brine. The organic layer was dried over $\mathrm{Na}_{2} \mathrm{SO}_{4}$, and concentrated under reduced pressure. The pure product was obtained by preparative tlc.

18) The yield of $\mathbf{2}$ under air was lower than that under oxygen atmosphere.

19) $2 \%$ of 4-chlorobenzoic acid was obtained and $9 \%$ of 5 was recovered.

20) The yield of $\mathbf{2}$ under discontinuous of irradiation was much lower than that under the usual conditions.

21) Minisci F., Porta O., Recupero F., Punta C., Gambarotti C., Pierini M., Galimberti L., Synlett, 2004, 2203-2205 (2004). 